Exci tat i on-power-dependent spect $r$ al shift i $n$ phot ol umi nescence i $n$ dye mol ecul es i $n$ strongl y scattering opt i cal medi a

\begin{tabular}{|l|l|}
\hline 著者 & $\begin{array}{l}\text { Tal ukder Am nul, Tot suka Kouki, Nat sumbt o M, } \\
\text { Tom t a Nakot o }\end{array}$ \\
\hline $\begin{array}{l}\text { j our nal or } \\
\text { publ i cat i on t i t l e }\end{array}$ & Physi cal Revi ew B \\
\hline vol une & 59 \\
\hline nunber & 1 \\
\hline page range & $50-53$ \\
\hline year & $1999-01$ \\
\hline 出版者 & Amer i can Physi cal Soci et y \\
\hline 権利 & (c) 1999 The Aner i can Physi cal Soci et y \\
\hline URL & ht t p: //hdl . handl e. net /10297/580 \\
\hline
\end{tabular}




\title{
Excitation-power-dependent spectral shift in photoluminescence in dye molecules in strongly scattering optical media
}

\author{
K. Totsuka, M. A. I. Talukder, M. Matsumoto, and M. Tomita \\ Department of Physics, Faculty of Science, Shizuoka University, 836 Ohya, Shizouka, 422 Japan \\ (Received 20 April 1998; revised manuscript received 28 September 1998)
}

\begin{abstract}
Photoluminescence spectra and the reflectivity of incident light were examined in dye molecules in strongly scattering media. With increasing incident light intensity, the spectra show red- and blueshifts depending on the geometry of experiments, and the reflectivity increases nonlinearly. These excitation-power-dependent spectral shifts and nonlinear reflectivity are well explained when we consider saturation absorption in the excitation process and the reabsorption in the luminescence process in dyes. [S0163-1829(99)14701-5]
\end{abstract}

Optical phenomena in strongly scattering media have been attracting much interest. In such systems light undergoes multiple scattering and travels a long path length in the medium. The coherent backscattering peak which arises from a constructive interference of time-reversed paths of multiply scattered light in the medium is a precursor of Anderson localization. ${ }^{1,2}$ Ahmed et al. ${ }^{3}$ reported the luminescence from dyes in a scattering medium showed strong spectral shift owing to the reabsorption of luminescence light because the path length for the luminescence light increases considerably in strongly scattering medium.

Nonlinear optics in strongly scattering media is a challenging and potential field. The long path length for the light resulting from multiple scattering leads to strong coupling between the light and material and results in nonlinear phenomena. Absorption is always a negative factor to observe the multiple-scattering effect because it terminates the long paths while amplification could enhance the multiplescattering effect. Wiersma et al. reported the narrowing of the coherent backscattering peak in Ti:sapphire powder with a large gain. ${ }^{4}$ Another interesting phenomenon in a strongly scattering medium may be the laser action. ${ }^{5}$ Lawandy et al. succeeded in observing such a laser action in dyes embedded in $\mathrm{TiO}_{2}$ microparticles solution in methanol. ${ }^{6}$ Subsequently the spectral, ${ }^{7,8}$ temporal, ${ }^{9}$ and spatial $^{10}$ properties of this emission, coinciding with some theoretical investigations $^{11-13}$ on the laser action in the scattering medium also have been reported.

In this paper, we investigate the nonlinear excitation process in dyes in strongly scattering media from the photoluminescence and the reflectivity measurements. With increasing excitation pulse energy, we observed the nonlinear reflectivity of the incident light as well as the spectral redand blueshifts for reflection- and transmission-type experiments, respectively. We explain our results with a combination of saturation absorption in the excitation process and reabsorption in the luminescence process.

For the spectral measurements, colloidal solutions containing sulforhodamine 640 dye in water and polystyrene spheres were used. The concentration of dye was from $1 \times 10^{-3} \mathrm{M}$ to $1 \times 10^{-6} \mathrm{M}$. Polystyrene spheres with a diameter of $0.45 \mu \mathrm{m}$ were purchased from Sekisui Kougyou Kagaku. ${ }^{14}$ In all cases of experiments, the density of polystyrene spheres was $6 \%$. The sample was pumped by linearly polarized $532 \mathrm{~nm}$ laser pulses from a frequency doubled $Q$-switched $\mathrm{Nd}^{3+}$ YAG laser with a pulse width of $8 \mathrm{~ns}$ and a pulse repetition rate of $5 \mathrm{~Hz}$. The excitation pulse energy was from 0.0001 to $4 \mathrm{~mJ}$. We performed reflection- and transmission-type experiments. In the reflection-type experiment, the sample cell was cylindrical with a diameter of 11 $\mathrm{mm}$ and the luminescence spectrum from the same surface on which the excitation light incidents was measured. For the transmission-type experiment, the sample cell was a thin $\left(0.1 \mathrm{~mm} \times 10^{2} \mathrm{~mm}^{2}\right)$ one and the luminescence spectrum from the opposite surface was studied. The excitation spot size on sample surface was $100 \mu \mathrm{m}$ and $2 \mathrm{~mm}$ for reflectionand transmission-type experiments, respectively. The emitted luminescence from the sample was led to a $25 \mathrm{~cm}$ polychromater and detected by a CCD camera. The spectral resolution was $0.14 \mathrm{~nm}$.

The reflectivity of the incident $532 \mathrm{~nm}$ excitation light was also measured in the reflection-type experiment. For the reflectivity measurements, colloidal solutions containing rhodamine 640 dye in methanol and $\mathrm{TiO}_{2}$ particles were used. The diameter of the $\mathrm{TiO}_{2}$ particle was $0.28 \mu \mathrm{m}$ and the particle density was $1 \times 10^{12} \mathrm{~cm}^{-3}$. To obtain the reflectivity, the reflected excitation light intensity from the sample with dye was normalized by the reflected excitation light intensity from the sample without dye.

Figure 1 shows the luminescence spectra for different excitation pulse energies in reflection-type experiments. The observed spectrum is redshifted with excitation pulse energy. Figure 2 shows the wavelength of luminescence peak with the excitation pulse energy for four different dye concentrations. At the concentration of $1 \times 10^{-6} \mathrm{M}$ the wavelength of the luminescence peak is almost constant. When the concentration is increased to $1 \times 10^{-3} \mathrm{M}$ the luminescence peak starts to shift toward the long-wavelength region at the pulse energy around $0.01 \mathrm{~mJ}$. Figure 3 shows the luminescence spectra for the transmission-type experiment. In this case, the wavelength of luminescence peak is blueshifted with increasing excitation pulse energy. Figure 4 shows the reflectivity of the incident excitation light of $532 \mathrm{~nm}$ with excitation pulse energy for two different dye concentration. It is seen that the reflectivity is almost constant at a concentration of $1 \times 10^{-5} \mathrm{M}$. When the concentration is increased to $1 \times 10^{-3} \mathrm{M}$ the reflectivity starts to increase at a pulse energy of about $0.4 \mathrm{~mJ}$. 


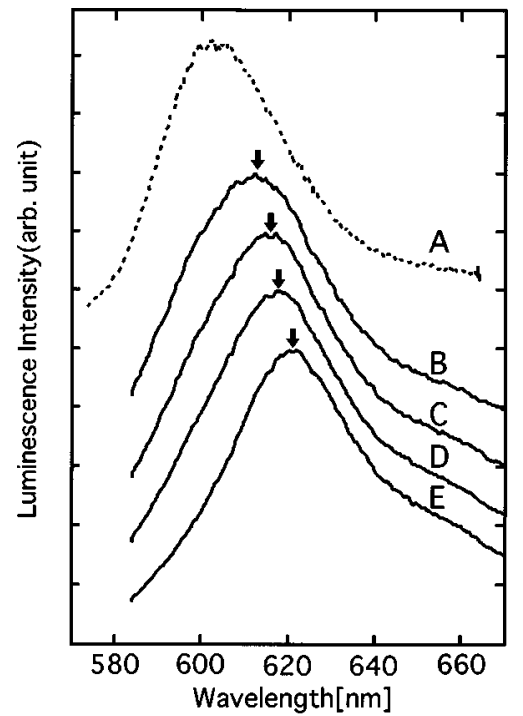

FIG. 1. Luminescence spectra in the reflection experiment from a colloidal solution containing $1 \times 10^{-3} \mathrm{M}$ sulforhodamine 640 in water and polystyrene spheres for different excitation pulse energy. (B) $0.0001(\mathrm{~mJ})$; (C) $0.001(\mathrm{~mJ})$; (D) $0.01(\mathrm{~mJ})$, and (E) 0.1 $(\mathrm{mJ})$. (A) is the luminescence spectrum in a pure dye solution in water of molecular density $1 \times 10^{-7} \mathrm{M}$. Arrows indicate the luminescence peak.

We consider that the spectral shifts and the nonlinear reflectivity are caused by the change in the spatial distribution of the excited-state population owing to saturation absorption in the excitation process of dyes. The penetration depth of the excitation light increases with the excitation pulse energy and the dye molecules in the deeper region are excited. In the reflection-type experiment, the luminescence coming from the deeper region is strongly reabsorbed before going out of the sample and the observed spectra show a large redshift. In the transmission-type experiment, the traversed path length for luminescence becomes shorter with increasing excitation pulse energy and the spectrum shows a blueshift. The increase in reflectivity of excitation light can also be explained by the bleaching of dye for the excitation light owing to saturation absorption.

To get quantitative information we developed a theoretical model which furnishes the study of luminescence and

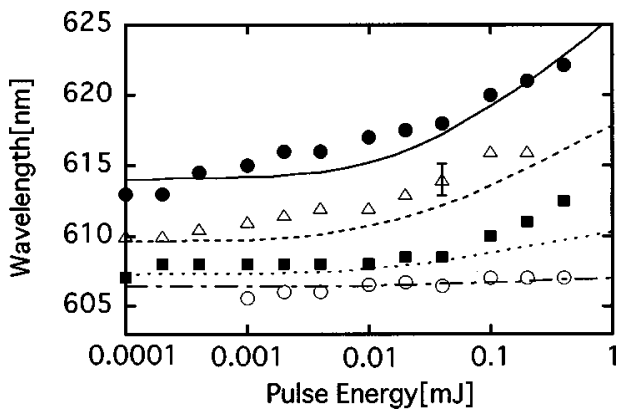

FIG. 2. Wavelength of the luminescence peak as a function of the excitation pulse energy for four different dye concentrations,

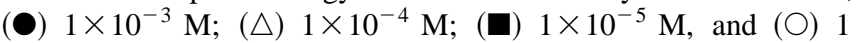
$\times 10^{-6}$ M. Solid, dashed, dotted, and dot-dashed lines are calculated curves of the wavelength for the luminescence peak for 1 $\times 10^{-3} \mathrm{M}, 1 \times 10^{-4} \mathrm{M}, 1 \times 10^{-5} \mathrm{M}$, and $1 \times 10^{-6} \mathrm{M}$, respectively.

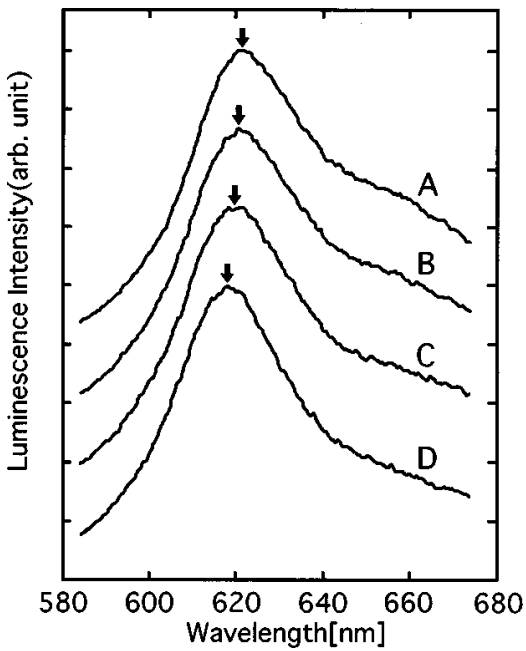

FIG. 3. Luminescence spectra in the transmission experiments from the colloidal solution containing $1 \times 10^{-3} \mathrm{M}$ sulforhodamine 640 in water and polystyrene spheres for the excitation pulse energy. (A) $0.001(\mathrm{~mJ})$; (B) $0.1(\mathrm{~mJ})$; (C) $1(\mathrm{~mJ})$, and (D) $4(\mathrm{~mJ})$. The sample used is the same as for Fig. 1 .

reflectivity in the presence of saturation absorption. We consider the two-level system consisting of the ground state $S_{0}$ and the excited state $S_{1}$ in the singlet levels for dyes. Since the nonradiative decay rate within $S_{0}$ and $S_{1}$ manifold is very fast, we assume that the population of $S_{0}$ and $S_{1}$ are at its lowest sublevel and higher sublevels are empty. Then, the excited molecules emit light through spontaneous emission from the lowest sublevel of $S_{1}$ to the $S_{0}$. Considering the dye molecular density is $N$ and the populations of dye molecule in $S_{0}$ and $S_{1}$ states are $N_{0}$ and $N_{1}$, respectively, one can write the rate equation for a two-level system as

$$
\frac{d N_{1}(\mathbf{r}, t)}{d t}=\sigma_{a}\left(\lambda_{e}\right) N_{0}(\mathbf{r}, t) I_{e}(\mathbf{r})-\frac{N_{1}(\mathbf{r}, t)}{\tau},
$$

where $N=N_{0}(\mathbf{r})+N_{1}(\mathbf{r})$. Here, $I_{e}(\mathbf{r})$ is the light intensity for the excitation light, $\sigma_{a}\left(\lambda_{e}\right)$ is the absorption cross section

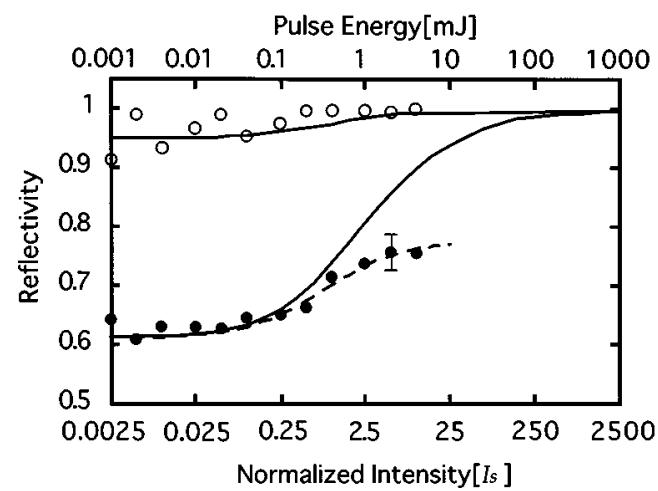

FIG. 4. Reflectivity of the excitation light as a function of the excitation pulse energy for two different dye concentrations, (O) $1 \times 10^{-3} \mathrm{M}$; (○) $1 \times 10^{-5} \mathrm{M}$. Solid and dashed lines are calculated curves of the reflectivity in the absence and in the presence of stimulated emission, respectively, as a function of the excitation pulse energy. 


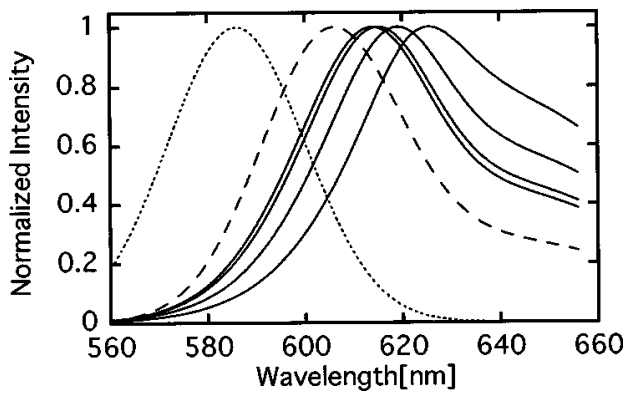

FIG. 5. Solid lines are calculated spectra for excitation pulse energies, $0.0001(\mathrm{~mJ}), 0.01(\mathrm{~mJ}), 0.1(\mathrm{~mJ})$, and $1(\mathrm{~mJ})$ consecutively from left to right for the colloidal solution of the 1 $\times 10^{-3} \mathrm{M}$ dye concentration. Dotted and dashed lines are the absorption and the luminescence spectra in the absence of reabsorption, respectively.

at excitation wavelength $\lambda_{e}$, and $\tau$ is the lifetime of the excited state. We consider that the region $z>0$ is filled with scattering medium with dyes. The stationary diffusion equations with absorption for excitation and luminescence light can be written as

$$
\begin{aligned}
& 0=\frac{l^{*}}{3} \nabla^{2} I_{e}(\mathbf{r})-\sigma_{a}\left(\lambda_{e}\right) N_{0}(\mathbf{r}) I_{e}(\mathbf{r}), \\
& 0=\frac{l^{*}}{3} \nabla^{2} I_{l}\left(\mathbf{r}, \lambda_{l}\right)-\sigma_{a}\left(\lambda_{l}\right) N I_{l}\left(\mathbf{r}, \lambda_{l}\right),
\end{aligned}
$$

where $I_{l}\left(\mathbf{r}, \lambda_{l}\right)$ is the light intensity for luminescence light at the wavelength $\lambda_{l}, \sigma_{a}\left(\lambda_{l}\right)$ is the absorption cross section at luminescence wavelength $\lambda_{l}$, and $l^{*}$ is the transport mean free path. We assume that $l^{*}$ is not dependent on the wavelength of light.

In the luminescence process, emitted light undergoes multiple scattering and travels in the sample with reabsorption by dye molecules. The absorption cross section at a shorter wavelength region is larger than the longer wavelength region in the dye solution used in our experiment. This results in the spectral shift. Since the quantum efficiency for sulforhodamine 640 in this sample is low, we neglect the luminescence emitted by the reabsorption. The incident excitation light from the region, $z<0$ excites the dye molecules in the sample and the luminescence intensity going out from the plane, $z=0$ is calculated in the reflection configuration. Solid lines in Fig. 5 show the calculated spectra for four different pulse energies in the reflection configuration. The parameters used in the simulation for the spectral measurement are as follows. $l^{*}$ is $31 \mu \mathrm{m}$, the absorption cross section $\sigma_{a}\left(\lambda_{e}\right)$ for $532 \mathrm{~nm}$ radiation is $0.96 \times 10^{-20} \mathrm{~m}^{2}$, and the excited-state lifetime $\tau$ is $0.145 \mathrm{~ns}$. The saturation intensity $I_{s}$ $=1 /\left[\sigma_{a}\left(\lambda_{e}\right) \tau\right]$ is calculated from the values of $\sigma_{a}\left(\lambda_{e}\right)$ and $\tau$, and is $7.2 \times 10^{29} \mathrm{~m}^{-2} \mathrm{~s}^{-1}$, which corresponds to $0.017 \mathrm{~mJ}$ of the excitation pulse energy. $\sigma_{a}\left(\lambda_{l}\right)$ and the luminescence spectrum are plotted with dotted and dashed lines in Fig. 5, respectively, which are extrapolated from absorptance and luminescence experiments in an aqueous solution of sulforhodamine 640. From these spectra in Fig. 5, we obtained the wavelength of the luminescence peak. The calculated wave- length of the luminescence peak is plotted in Fig. 2 as a function of the excitation energy and shows good agreement with experimental results.

The reflectivity of the excitation light could also be calculated using Eqs. (1) and (2). In Fig. 4 solid lines represent the calculated reflectivity for $1 \times 10^{-5} \mathrm{M}$ and $1 \times 10^{-3} \mathrm{M}$ samples. The parameters used in the simulation for reflectivity measurement are as follows. $l^{*}$ is $7 \mu \mathrm{m}$ for $\mathrm{TiO}_{2}$ particles of $0.28 \mu \mathrm{m}$ in diameter with a particle density of 1 $\times 10^{12} \mathrm{~cm}^{-3}$, the absorption cross section $\sigma_{a}\left(\lambda_{e}\right)$ for 532 $\mathrm{nm}$ radiation is $1.2 \times 10^{-20} \mathrm{~m}^{2}$, and the excited-state lifetime $\tau$ is $1.9 \mathrm{~ns}$. The saturation intensity $I_{s}$ is $4.4 \times 10^{28} \mathrm{~m}^{-2} \mathrm{~s}^{-1}$ which corresponds to $0.4 \mathrm{~mJ}$ of the excitation pulse energy with the excitation light beam diameter of $2 \mathrm{~mm}$. It starts to increase at the saturation intensity, and then converges to 1 at an excitation region far larger than the saturation intensity.

The experimental results shown in Fig. 4 for the 1 $\times 10^{-5} \mathrm{M}$ sample are in good agreement with the theoretical one, while the experimentally obtained reflectivity for the 1 $\times 10^{-3} \mathrm{M}$ sample is small compared with the theoretical curve in the strong excitation region. We explain this difference in terms of the stimulated emission of the luminescence light. In an excitation intensity more than the saturation intensity, we observed the spectral collapse in the luminescence spectrum in the methanol sample like many other experiments carried out on scattering media. ${ }^{8-12}$ In the presence of stimulated emission, the rate equation for the excited-state population of dyes can be written as Eq. (1) with an additional term $-\sigma_{s}\left(\lambda_{l}\right) N_{1}(z, t) I_{l}(z)$ on the right side. Here $\sigma_{s}\left(\lambda_{l}\right)$ is the stimulated emission cross section, and $I_{l}(z)$ is the luminescence light intensity. We assumed the stimulated emission cross section for luminescence light was independent of the wavelength and estimated to be 0.6 $\times 10^{-20} \mathrm{~m}^{2}$. The dashed line represents a similar calculation but with taking the stimulated emission term into account in Eq. (1). In the strong excitation region, the reflectivity expressed by the solid line is larger than the experimental result, whereas the dashed line is in good agreement with experimental result.

In our analyses we assumed that the reabsorption coefficients for the luminescence light from dyes are independent of the excitation power. This assumption is, however, not good for use in the actual sample. We also performed photoluminescence measurement using the thin sample cell in the reflection configuration. In this measurement (Fig. 3), a slight blueshift was observed with increasing the incident light intensity. This blueshift is in contrast to the redshift observed in the reflection configuration in a thick sample cell $11 \mathrm{~mm}$ in diameter and suggests that bleaching is actually taking place at the wavelength of the luminescence.

The saturation absorption may be a very important mechanism in the laser action in the strongly scattering medium. Berger et al. ${ }^{13}$ simulated that the luminescence spectra of rhodamine 640 in random scattering medium in the presence of stimulated emission show the redshift followed by the blueshift in the very strong excitation region. They explained that these spectral features result from the interplay of amplification and reabsorption of the luminescence light. This blueshift could be the same effect as we observed for the thin sample in the reflection measurement. It may, however, not appear clearly owing to the effect of stimulated 
emission. It should be noted that the main difference between previous reports ${ }^{6-8}$ and the present one is that in our case dye molecules are dissolved in water. The radiative efficiency of dyes in water is small (about 7\%) because dye molecules form a dimer that does not luminesce in water. This would be the reason why we were able to observe systematic spectral red- and blueshifts in the luminescence spectra and explained it quantitatively. The laser action in strongly scattering medium is a very complicated phenomenon that is affected by many factors. In our sample, we can selectively examine the excitation process without the amplifying process by the stimulated emission. When the solvent was replaced with methanol, we had easily observed the spectral collapse with increasing excitation pulse energy before the systematic spectral shift occurred.

We observed the spectral red- and blueshifts in the luminescence and the nonlinear reflectivity in the excitation light from strongly scattering media with dyes. These phenomena are attributed to the change in the distribution of the population of the excited state owing to the saturation absorption in dye molecules. Strong scatterers confine light in restricted spatial regions and increase the interaction between light and materials. This spatial confinement may be useful in observing the nonlinear optical effect as well as in the design of optical devices.
${ }^{1}$ M. P. Van Albada and A. Lagendijk, Phys. Rev. Lett. 55, 2692 (1985).

${ }^{2}$ P.-E. Wolf and G. Maret, Phys. Rev. Lett. 55, 2696 (1985).

${ }^{3}$ S. A. Ahmed, Z.-W. Zang, K. M. Yoo, M. A. Ali, and R. R. Alfano, Appl. Opt. 33, 2746 (1994).

${ }^{4}$ D. S. Wiersma, M. P. van Albada, and A. Lagendijk, Phys. Rev. Lett. 75, 1739 (1995).

${ }^{5}$ R. V. Ambartsumyan, P. G. Kryukov, and V. S. Letkhov, Sov. Phys. JETP 24, 1129 (1967).

${ }^{6}$ N. M. Lawandy, R. M. Balachandram, A. S. Gomes, and E. Sauvain, Nature (London) 368, 436 (1994).

${ }^{7}$ W. Zhang, N. Cue, and K. M. Yoo, Opt. Lett. 20, 961 (1995).
${ }^{8}$ G. Beckering, S. J. Zilker, and D. Haarer, Opt. Lett. 22, 1427 (1997).

${ }^{9}$ M. Siddique, R. R. Alfano, G. A. Berger, M. Kempe, and A. Z. Genack, Opt. Lett. 21, 450 (1996).

${ }^{10}$ D. S. Wiersma, M. P. van Albada, and A. Lagendijk, Nature (London) 373, 203 (1995); N. M. Lawandy and R. M. Balachandran, ibid. 373, 204 (1995).

${ }^{11}$ S. John and G. Pang, Phys. Rev. A 54, 3642 (1996).

${ }^{12}$ R. M. Balachandran, N. M. Lawandy, and J. A. Moon, Opt. Lett. 22, 319 (1997).

${ }^{13}$ G. A. Berger, M. Kempe, and A. Z. Genack, Phys. Rev. E 56, 6118 (1997).

${ }^{14}$ T. Kuge, Funct. Mater. 2, 58 (1983). 